\title{
Atmospheric effects in infrared thermography
}

by P.PREGOWSKI (*)

\author{
(*) PIRS -Preggowski Infrared Services, P.O.Box 2, 00-908 Warsaw, Poland
}

\begin{abstract}
For some sort of interpretation of thermographic works, as well as for devices designing and for planning of investigations it is recommended to considerate not only transmission but also emission effects of the atmosphere. Basing on modelling and spectral calculations some of examples there are shown the same as some results off long-term measurements of the reflected signals going from the atmosphere.
\end{abstract}

\section{Introduction}

Total error in the temperature determination using infrared thermographic devices may be considered to be a sum of the errors caused by instrument and calibration, emissivity and atmosphere. Emissivity anci atmospheric errors are often most important.

Real emitters partially reflect radiation. Their signals consisted of several components e.g. radiances of the sun, cloud or sky. The earth's atmosphere causes simultaneously to attenuate the radiation. The main elements of the atmospheric influences are:

- absorption by the molecular constituents (e.g. water vapor, carbon dioxide, nitrous oxide);

- emission from constituents as above and from particle of matter;

- scattering of radiation out of the path, by molecules and particles;

- scattering of unwanted radiation these components into the optical path;

- refraction, both of them out the path and into it.

Additionally turbulences cin affect all of these elements. it apear to be the major : omplex factor many of infrared analyses. We can find many of similar problems also taking to consideration industrial thermografic investigations, e.g. when hot old cold gases and aerosols exists.

For some of infrared thermal applications, e.g. military or environmental particulary important are knowledge and accurate analyses of the atmospheric effects. Many of data and modelling or calculation procedures are available now in open literature. The aim of this short presentation is to signalize some of the atmospheric influences based on have been done calculations and investigations $[1,2]$.

\section{Considerations}

Since the response of the infrared detector and processing unit are assumed to be linear with the effective radiance, output signal of the infrared device may be consider as proportional to radiance $L$ according to equation (1). For simplicity we will use effective radiances what means that adequate coefficients of the emission and of the atmosphere transmission are covered inside these radiances. 
http://dx.doi.org/10.21611/qirt.1992.053

$$
\begin{aligned}
L= & \int_{\lambda}^{\lambda}[\{\operatorname{Loe}(\lambda, \ldots)+\operatorname{Lor}(\lambda, \ldots)+\operatorname{La}(\lambda, \ldots)\} S(\lambda, \ldots)+ \\
& \left.+\operatorname{Lint}(\lambda, \ldots) S^{\prime}(\lambda, \ldots)\right] d \lambda
\end{aligned}
$$

where $\lambda_{1}$ to $\lambda_{2}$ spectral band pass of the system,

$L_{o e}(\lambda, \ldots)$ effective spectral radiance of the object emission,

$\operatorname{Lor}(\lambda, \ldots)$ effective spectral background radiance reflected from the object surface,

$\mathrm{La}(\boldsymbol{\lambda}, \ldots)$ effective spectral radiance due to atmospheric emission and scatered energy,

$\operatorname{Lint}(\lambda, \ldots)$ effective spectral radiance due to device internal emission,

$S(\lambda, \ldots)$ relative sensing device response,

$S^{\prime}(\lambda, \ldots)$ is the relative sensing device response for signals due to internal emission of the device.

Detected thermal signals, therefore are varying with the type of the detector, spectral band pass, the surface emittance and atmospheric conditions. Quantitative, spectral-energetic analyses and calculations provide many information being covered during practical measurements.

It is practical to describe influence of the atmospheric path between object of investigation and infrared detector by effective transmission factor $\boldsymbol{r}_{\mathrm{a}, \mathrm{ef}}$ :

$$
\tau_{\mathrm{a}, \mathrm{ef}}=\frac{\int^{\lambda} L(\lambda, T, \ldots) \tau{ }_{\mathrm{a}, \lambda}^{\lambda} S(\lambda, \ldots) d \lambda}{\int_{\lambda}^{\lambda} L(\lambda, T . \ldots) S(\lambda, \ldots) d \lambda}
$$

To differentiate the changes of $\boldsymbol{r}_{\mathrm{a}, \mathrm{ef}}$ as a function of chosen components e.g. the scanning angle $\boldsymbol{\Omega}_{\mathrm{p}}$ we used coefficient $k_{\mathrm{ra}, \mathrm{e}}\left(\boldsymbol{\Omega}_{\mathrm{p}}\right)$ of relative changes of $\boldsymbol{r}_{\mathrm{a}, \mathrm{e}}$ expressed below:

$$
k_{\tau_{a, e f}(\Omega p)}=\frac{\tau_{a, \text { ef }}\left(\Omega_{p}\right)}{\tau_{a, \text { ef }}\left(\Omega_{p}=0\right)}
$$

Basing on adequate modelling and computer calculations it is possible to look for lowering of mentioned artifacts by taking the advantage of the spectral contrast coefficient $C R_{1}$ : 
http://dx.doi.org/10.21611/qirt.1992.053

$$
C R_{\lambda}=\frac{L_{o, e}(\lambda, \ldots)-\left[L_{\circ, \mathrm{r}}(\lambda, \ldots)+L_{\mathrm{a}}(\lambda, \ldots)\right]}{L_{\circ, \mathrm{e}}(\lambda, \ldots)+\left[L_{o, \mathrm{r}}(\lambda, \ldots)+L_{\mathrm{a}}(\lambda, \ldots)\right]}
$$

\section{Results}

Below, some examples of the different kind of atmospheric influences are discussed assuming the same typical measuring conditions:

- single, large, lambertian type object with emissivity coefficient $\epsilon=0.8$ (not spectraly variable) and temperature $288 \mathrm{~K}$ exist near the blackbody background with the temperature $283 \mathrm{~K}$; are characterized by :

-air temperature $283 \mathrm{~K}$, relative air humidity $85 \%$, air pressure $1000 \mathrm{hPa}$, continental environment, without haze and rain.

Figure 1 shows the spectral distribution of the effective radiances of individual four signals just before device optical transformation : a as total radiance being a sum of such radiances as: $b$ for self radiation of object, $c$ for radiation of atmosphere, $d$ for reflected radiation of background.

Crossed parts in figure 1 present spectral bands in which self emission of the object is dominant. It is evident that truly good window being loked for obtain high level of wanted information is within the relatively wide range 3-5 $\mu \mathrm{m}$. For small differences of the atmospherio and object temperatures atmospheric emission is the significient part of entrance signal.

The same problem is shown in figure 2 for long wave infrared devices. Because atmospherlo absorption in this wavelength range is much more regular and low, the above mentioned problems seem to be not so serious as for shorter IR waves.

- Basing on equation (4) and assuming that only self emission of object surface carries interesting information $C R_{1}$ course is shown in figure 3. Such types of analyses are useful for device desigring as well as for proper planning works for the thernıographic investigations.

Of course, narrowing of the range of spectral sensitivity causes lowering of total detected signal. Their ratio to the internal noise of the instrument will be worse. Now, many methods of compensation are possible due to the popularity of digitalization and memorization.

Differences caused by atmosphere are especially important for wide-angle and long range operations. It is because the length of the radiation path varies with the scanning angle. Such examples are shown at figure 4.

The last figures 5 and 6 are examples of long term examinations have been done in the Optoelectronics Institute of the Warsaw Military Academy of Technology (MAT).

We have asked the question what are the main quantitative connections between state of the sky (weather conditions) and spatial position of object surface to the IR device direction of view?

Because it is work with the truly variable medium, we assumed cyclic measurements to obtain statistical data [3]. Only differences of equivalent temperature relative to zenith are presented, here. Absolute temperatures were different. Measurements have been done in 8-13 $\mu \mathrm{m}$ range. Figures were taken for object being $45 \mathrm{deg}$. inclined to the direction of observation and to zenith, simultaneously. This probe was rotated right and left from the zenith direction in the range of $+60 \mathrm{deg}$. to $-60 \mathrm{deg}$. The directional $45 \mathrm{deg}$. inclined emission coefficient was measured as 0.59. Circular surface being measured was inclined to North on $150 \mathrm{deg}$ Meteorological parameters were noted in the time of measurements (autumn and winter). 


\section{http://dx.doi.org/10.21611/qirt.1992.053}

Influences of the surface and air temperature, relative humidity, wind speed and direction, range of visibility (figure 5 , range $9 \mathrm{~km}$ and $0.9 \mathrm{~km}$ ), altitude and type of clouds (figure 6 , low altitude : stratus, cumulus, stratocumulus; medium altitude: alto stratus, alto cumulus and cirrus) were measured.

Reflected signals as a function of visibility for inclinations about $40 \mathrm{deg}$. stop the increasing character and lowered. Very near charts we obtained for other parametrs. Minimal dependance as a function of zeniyh angle we noted for: rainfall (in opposite to clean sky), very high humidity (in opposite to low level) and for fully cloudiness in opposite to non cloudy sky.

\section{Conclusions}

Some of factors determining the IR thermal investigation have been discussed together with the effects of the air path throug which the objects are measured. Proper understanding these factors is need for many of infrared applications in open air. It is possible to meet similar effects taking to consideration some of industrial disturbing mediums.

\section{Acknowledgments}

It is a pleasure to acknowledge the help of H.Polakowski, H.Bogalecki and H.Madira from MAT.

\section{REFERENCES}

[1] PREGOWSKI (P.).- The analyses of radiant signal marks and their influence for the thermographic devices designing. Doctor thesis, MAT, 1985.

[2] PREGOWSKI (P.).- Modelling for CAD analyses of telethermodetection problems. Metrologia i Systemy Pomiarowe, Conference proceedings, CPBP 02.20, Warsaw, 1991, p.127-136. 


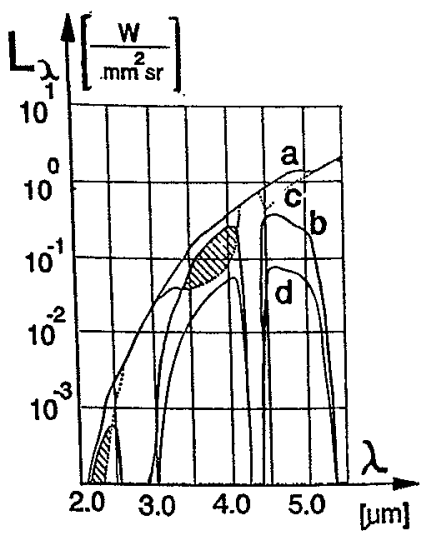

Fig.1. - Radiative properties of the entrance signals

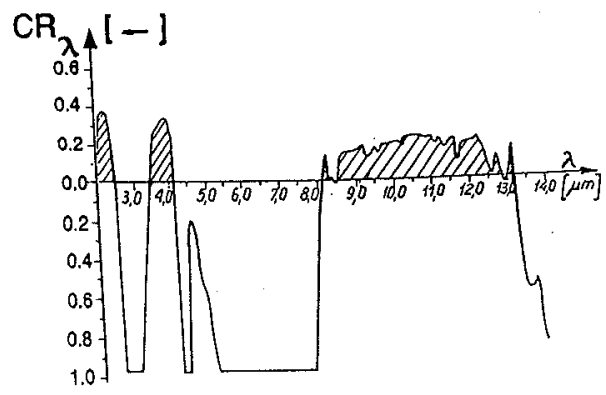

Fig.3. - Spectral distribution of the radiances contrast coefficient



Fig.5. - Reflected signals as a function of zenith angle and air visibility

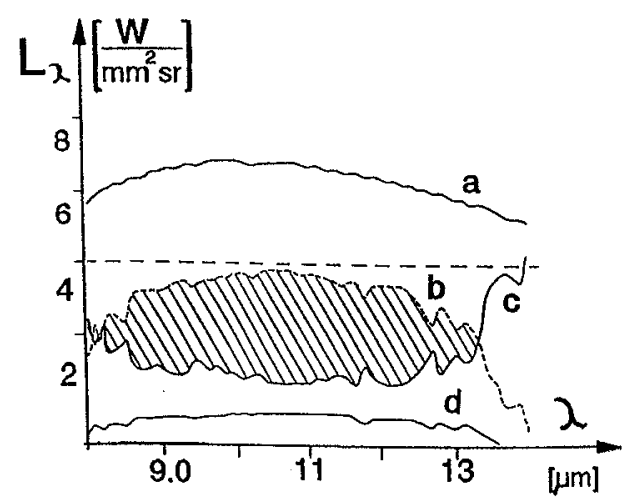

Fig.2. - Radiative properties of the entrance signals

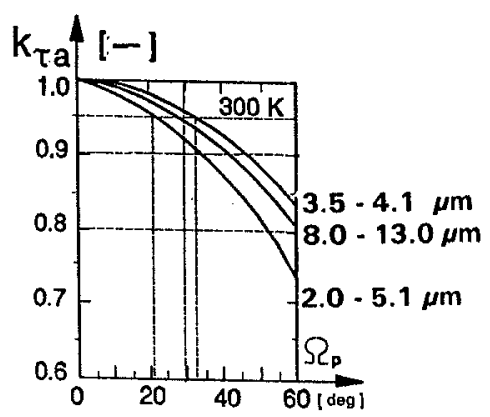

Fig.4. - Relative changes of the effective atmosphere transmission factor



Fig.6. - Reflected signals as a function of zenith angle and types of clouds 